\title{
IDENTIFICAÇÃO POR MICROSCOPIA RAMAN DE PIGMENTOS DA PINTURA A ÓLEO "RETRATO DE MURILO MENDES” DE CÂNDIDO PORTINARI
}

Luiz Fernando Cappa de Oliveira*

Departamento de Química - Instituto de Ciências Exatas - Universidade Federal de Juiz de Fora - 36036-330 - Juiz de Fora - MG Josefina del Carmen Renata Prieto Boscán

Centro de Estudos Murilo Mendes - Universidade Federal de Juiz de Fora - Av. Barão do Rio Branco 3372 - $36025-020$ -

Juiz de Fora - MG

Paulo Sérgio Santos e Márcia Laudelina Arruda Temperini

Instituto de Química - Universidade de São Paulo - CP 26077 - 05599 -970 - São Paulo - SP

Recebido em 10/4/97; aceito em 7/8/97

\begin{abstract}
IDENTIFICATION OF PIGMENTS FROM CANDIDO PORTINARI'S OIL PAINTING "PORTRAIT OF MURILO MENDES" BY RAMAN MICROSCOPY. In the present work "ex situ"Raman microscopy was employed in the identification of some of the pigments present in the painting "Portrait of Murilo Mendes" by Cândido Portinari. In the investigated samples it was possible to identify unambiguously two pigments: Prussian blue and lapis-lazuli (or its synthetic analogue, ultramarine blue) - the former is observed together with organic substances, possibly used as dispersants, what suggests a further use of a mass for recover the painting.
\end{abstract}

Keywords: Raman microscopy; inorganic pigments; Cândido Portinari.

\section{INTRODUÇÃO}

A microscopia Raman tem se mostrado nos últimos anos uma técnica analítica poderosa para determinação qualitativa de pigmentos utilizados em obras de arte, sendo uma técnica não destrutiva que combina sensibilidade e especificidade, imune a interferências (como por exemplo fluorescência), podendo inclusive ser aplicada in situ nas amostras estudadas ${ }^{1,2}$. A utilização do efeito Raman ressonante, no qual utiliza-se como fonte de excitação uma radiação eletromagnética com comprimento de onda no qual o pigmento absorve, torna ainda mais seletiva a técnica.

São vários os objetivos que justificam o estudo dos pigmentos em uma obra de arte $^{1}$ :

- caracterização: quais foram os pigmentos, simples ou combinados, usados pelo artista;

- restauração e conservação da obra: através do conhecimento dos pigmentos utilizados torna-se possível restaurar áreas danificadas, fazendo uso do material original;

- datação e autenticação: considerando-se que o uso de determinados pigmentos pode indicar a data ou época de utilização de tais espécies, é possível ter uma idéia do período de confecção do trabalho, bem como de sua autentificação.

Neste trabalho em particular a técnica de microscopia Raman foi utilizada para a identificação de alguns pigmentos da pintura a óleo "Retrato de Murilo Mendes ", efetuado por Cândido Portinari e datado do princípio da década de trinta (1933 ou 1935, não há precisão da data na obra). Esta obra pertence ao acervo do Centro de Estudos Murilo Mendes, da Universidade Federal de Juiz de Fora.

Como objetivo principal do estudo encontra-se a identificação de pigmentos originais usados por Portinari, investigando regiões da obra onde foi possível obter-se amostras, em virtude de apresentar lacunas ou perda de estratos pictóricos, possibilitando assim a retirada das mesmas para análise ex situ. Como será discutido mais adiante, foi possível também investigar regiões da obra

E-mail: pimenta@química.ufjf.br. que apresentam materiais indicativos de um possível emassamento, ou seja, denotando intervenções ou restaurações posteriores. De nosso conhecimento, este é o primeiro trabalho de identificação dos pigmentos usados em uma obra de Cândido Portinari, um dos expoentes da pintura brasileira.

\section{EXPERIMENTAL}

As amostras para microscopia Raman foram obtidas através da raspagem da tinta superficial do quadro, por praticamente toda a borda do mesmo. Os espectros Raman foram obtidos em um instrumento Renishaw Raman Imaging Microscope System 3000 , equipado com microscópio com capacidade de aumento de até oitenta vezes, sendo a detecção efetuada através de espalhamento da luz monocromática a $180^{\circ}$ (retroespalhamento) em um detector multicanal. A radiação utilizada foi a linha $632,8 \mathrm{~nm}$ de um laser de $\mathrm{He}-\mathrm{Ne}$, refrigerado a ar, da SpectraPhysics, com potência na amostra da ca. $70 \mu \mathrm{W}$, e com diâmetro do feixe da ordem de $5 \mu \mathrm{m}$. Utilizou-se sistema de varredura, com tempo de aquisição de 10 segundos, com acúmulo de até 30 espectros, e resolução espectral da ordem de $5 \mathrm{~cm}^{-1}$.

\section{RESULTADOS E DISCUSSÃO}

Fotografias do quadro de Portinari, com iluminação natural e com luz ultravioleta, podem ser observadas na figura 1 , juntamente com os pontos marcados referentes às amostras investigadas por microscopia Raman. Nota-se na figura 1 que, sob luz ultravioleta, regiões da obra aparecem com maior ou menor intensidade, em virtude da presença de verniz que costumeiramente recobre tais obras, objetivando proteger a mesma. A falta de recobrimento do verniz torna mais visível as imperfeições da obra quando analisada com luz ultravioleta.

As amostras 1 e 2 referem-se a uma região do quadro que apresentou, sob luz ultravioleta, coloração diferente do restante do quadro. Esta diferença pode ser creditada a dois motivos diferentes: a falta do verniz utilizado pelo autor para proteção do quadro, ou então o mesmo pode ter sido posteriormente modificado através de um processo conhecido como emassamento, 


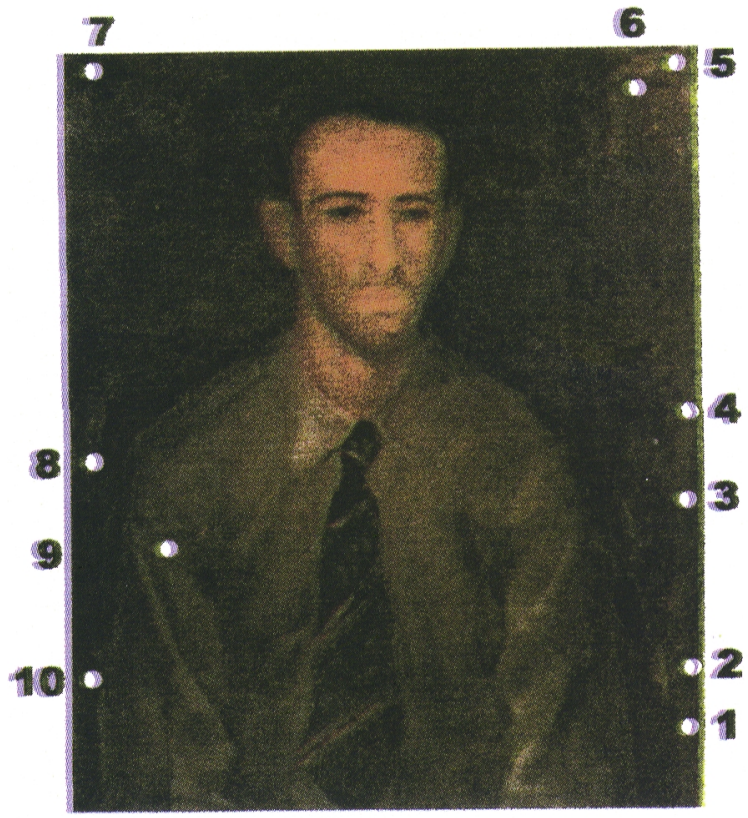

A

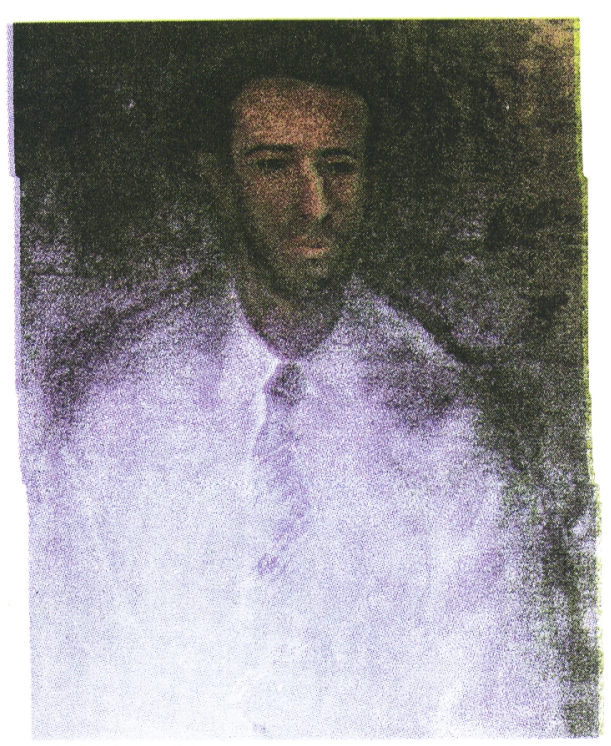

B

Figura 1. Fotografias do quadro a óleo "Retrato de Murilo Mendes", de Cândido Portinari, sob luz natural (A) e sob iluminação com lâmpada ultravioleta $(B)$. Os pontos marcados e enumerados referem-se às regiões investigadas neste trabalho.

no qual é feita a posteriori uma restauração da obra, embora sem nenhum conhecimento prévio do tipo de pigmento original utilizado pelo autor. Os espectros Raman obtidos para as duas amostras denotaram a existência de um mesmo tipo de pigmento, e um espectro Raman representativo pode ser observado na figura 2. Nas figuras $2 \mathrm{~A}$ e $2 \mathrm{~B}$ podem ser observados os espectros obtidos nas regiões de 400 a 1000 e de 1000 a $1800 \mathrm{~cm}^{-1}$, respectivamente, nos quais várias bandas estão presentes, indicando a presença de substância (ou substâncias) orgânica, cujos modos vibracionais são esperados nesta região. Entretanto, na figura $2 \mathrm{C}$ pode ser observado o espectro obtido para as mesmas amostras na região de 2000 a $2500 \mathrm{~cm}^{-1}$, onde percebe-se claramente a presença de modos vibracionais em 2090 e $2152 \mathrm{~cm}^{-1}$, típicos da vibração de estiramento da ligação tripla carbononitrogênio $\left(v_{\mathrm{C} \equiv N}\right)$ [3]. Como a coloração da tinta na região é azul, pode-se afirmar com certeza que o pigmento utilizado nessa região do quadro refere-se ao azul da Prússia, cuja espécie inorgânica presente é o complexo $\mathrm{Fe}_{2}\left[\mathrm{Fe}(\mathrm{CN})_{6}\right]$.
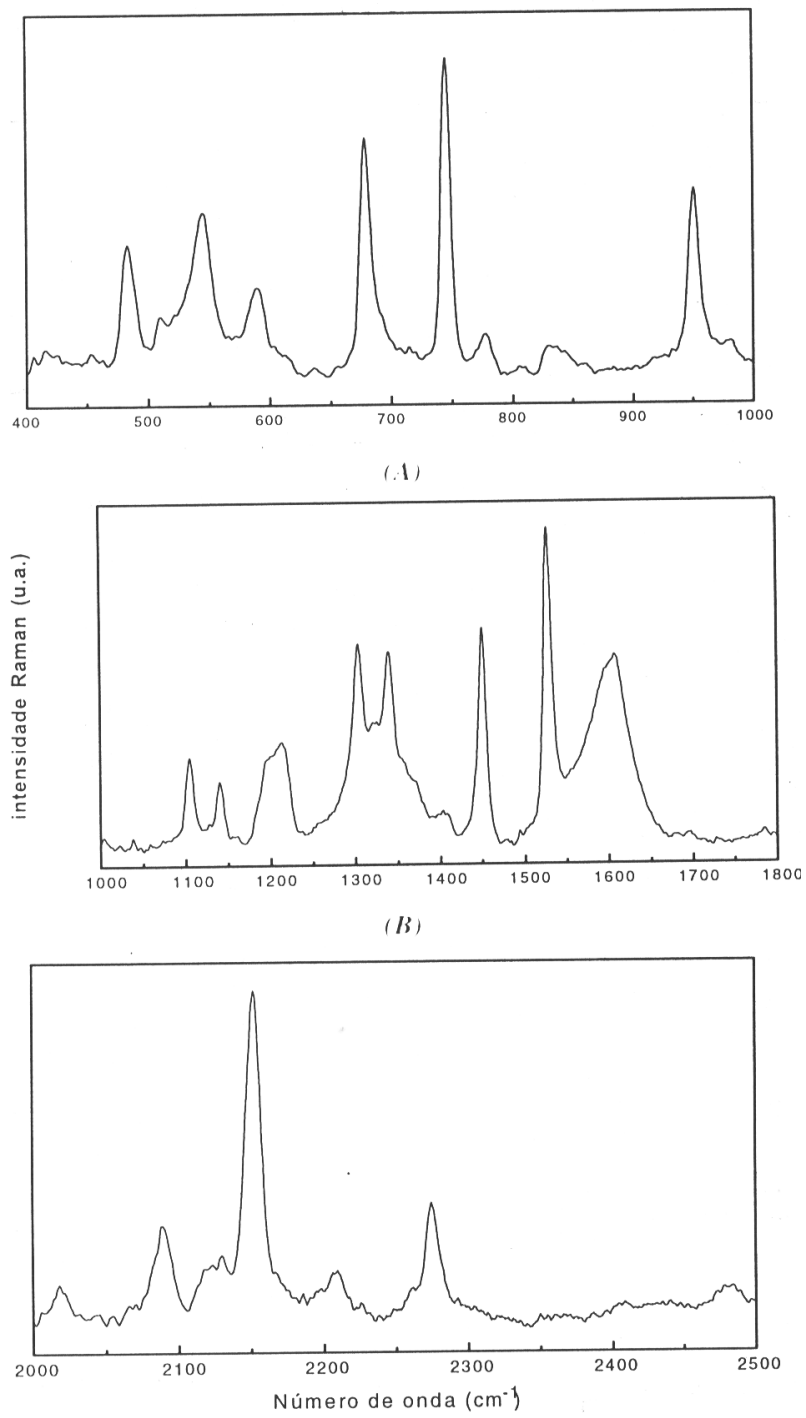

(C)

Figura 2. Espectros Raman típicos do emassamento recente feito na obra, obtidos das amostras 1 e 2 mostradas na Figura 1A, nas regióes de 400 a $1000 \mathrm{~cm}^{-1}$ (A), de 1000 a $1800 \mathrm{~cm}^{-1}$ (B) e de $2000 a_{2500 \mathrm{~cm}^{-1}}(C)$.

No processo de raspagem da amostra 2, foi possível obterse uma pequena amostra da base ainda com um depósito de coloração também azul. O espectro Raman dessa parte do quadro apresentou um padrão de bandas Raman totalmente distinto do obtido para o pigmento da superfície, e este espectro pode ser observado na figura 3 .

Como pode ser observado na figura 1 , com exceção da amostra 7 as demais foram obtidas de regiões da obra onde não há evidência de fluorescência, supondo-se assim que seriam referentes a pigmentos originais utilizados por Portinari na confecção do quadro. Não foi possível obter-se espectro Raman com excitação em 632,8 nm para a amostra 7, devido a um acentuado fundo de fluorescência. Um espectro Raman típico das demais amostras pode ser observado na figura 3 , ou seja, 


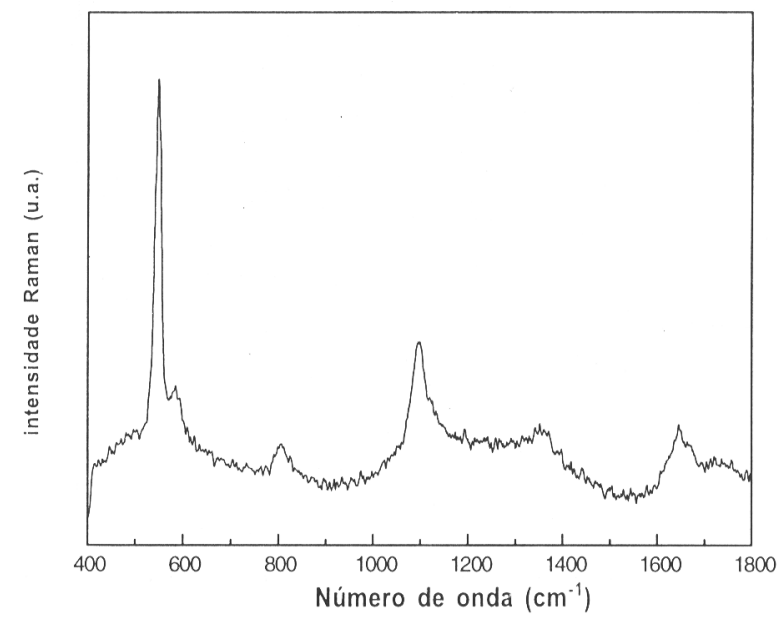

Figura 3. Espectro Raman típico referente às amostras 3 a 10 da Figura 1A, mostrando a frequência fundamental $\left(549 \mathrm{~cm}^{-1}\right)$ e as progressões obtidas com excitação em $632,8 \mathrm{~nm}$ para o lápis-lazuli.

as demais amostras estudadas mostraram o mesmo padrão espectral comparado à base da amostra 2 .

A figura 3 mostra três bandas Raman, respectivamente em 549, 1098 e $1649 \mathrm{~cm}^{-1}$. Comparando-se com dados da literatura $^{1,4-6}$, pode-se atribuir como pigmento básico utilizado por Portinari o lápis lazuli, um aluminossilicato (sodalita) que apresenta íons radicais $\mathrm{S}_{3}{ }^{-}$presos na estrutura, como dopantes. A transição eletrônica deste pigmento pode ser observada em $610 \mathrm{~nm}$, e tem como uma das bandas vibracionais mais intensas do espectro o modo conhecido como $v_{1}$, em $550 \mathrm{~cm}^{-1}$, referente ao estiramento totalmente simétrico da ligação enxofre-enxofre. Em outras palavras, foi observado o espectro Raman pré-ressonante do pigmento, hoje comercialmente conhecido como azul ultramarino (o equivalente sintético do lápis-lazuli). No espectro mostrado na figura 3 , o modo $v_{1}$ refere-se à banda Raman observada em $544 \mathrm{~cm}^{-1}$, e as outras duas bandas referem-se a progressões de $v_{1}$ (conhecidas como bandas harmônicas) comumente observadas no efeito Raman ressonante. O uso do azul ultramarino como pigmento por artistas impressionistas, tais como Monet e Renoir, é bem conhecido da literatura, e foi discutido do ponto de vista químico em um trabalho recente ${ }^{7}$.

Como termo de comparação, nas figuras 4 e 5 podem ser observados os espectros Raman do lápis-lazuli e do azul da Prússia, respectivamente, obtidos nas mesmas condições das amostras. O espectro do lápis-lazuli mineral mostra um fundo acentuado de fluorescência, o qual entretanto não mascara as bandas vibracionais, intensificadas pelo processo de ressonância. Pode-se notar a presença das três bandas Raman observadas também nas amostras do quadro, denotando assim a presença deste pigmento, contendo o grupo cromofórico $\mathrm{S}_{3}{ }^{-}$. As demais bandas observadas no espectro são devidas à matriz, provavelmente referentes a modos vibracionais do silicato. $\mathrm{Na}$ figura 5, o espectro do azul da Prússia mostra um pequeno número de bandas, relativas apenas aos modos metal-ligante (em torno de $500 \mathrm{~cm}^{-1}$ ) e estiramento $\mathrm{C} \equiv \mathrm{N}$, na região de 2150 $\mathrm{cm}^{-1}$. A observação deste modo vibracional nos espectros das amostras 1 e 2 confirma a utilização do azul da Prússia como pigmento utilizado a posteriori no emassamento da obra. Como pode ser visto nas figuras 2 e 5 , são observadas várias bandas vibracionais na região de estiramento $\mathrm{C} \equiv \mathrm{N}$; este fato é comum em compostos que apresentam ambientes químicos distintos no estado sólido, como o caso do íon complexo $\mathrm{Fe}(\mathrm{CN})_{6}{ }^{-}$, sendo que estes desdobramentos podem ser atribuídos a combinações com modos de rede ou ainda uma baixa simetria local.

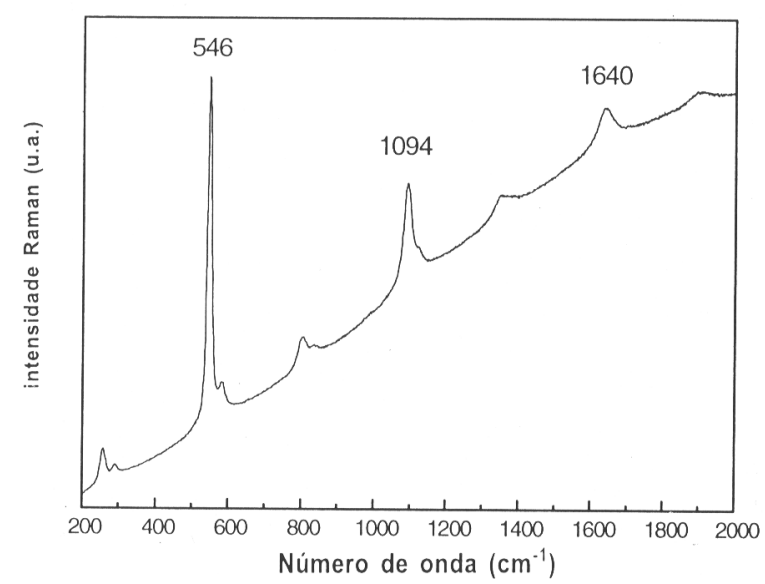

Figura 4. Espectro Raman de amostra de lápis-lazuli mineral, obtido com excitação em $632,8 \mathrm{~nm}$, com os modos vibracionais (fundamental e harmônicas) ressaltados.

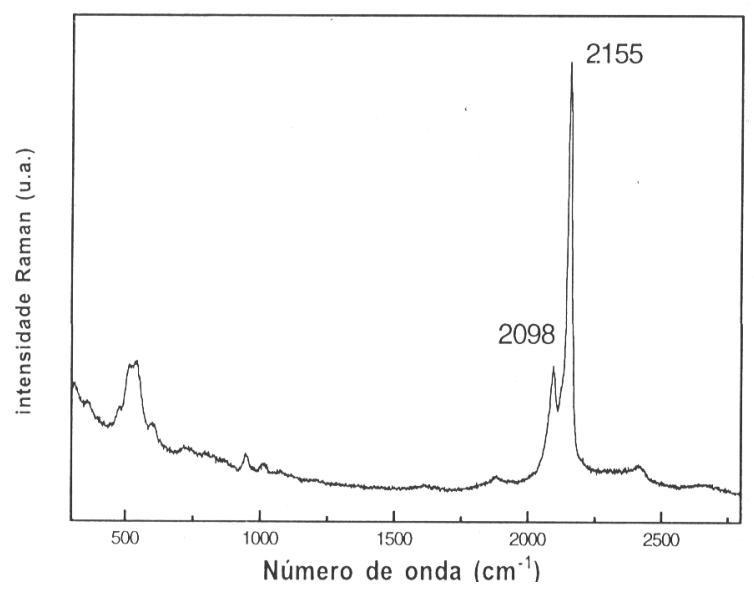

Figura 5. Espectro Raman do azul da Prússia puro, obtido com excitação em 632,8 nm, com os principais modos vibracionais ressaltados.

É interessante notar que no espectro da figura 3, característico de regiões originais do quadro de Portinari, não há a presença de bandas referentes a modos típicos de compostos de origem orgânica. Comparado com o espectro da figura 2, pode-se assim concluir que na tinta usada na região das amostras 1 e 2 ainda há a presença do dispersante orgânico usado juntamente com o pigmento. Cabe lembrar que tintas à base de óleo como dispersante apresentam uma vida média de aproximadamente 50 anos, isto é, cerca de 50 anos são necessários para a total evaporação do óleo ${ }^{8}$. Este fato é mais um suporte para a idéia de que realmente o quadro original foi emassado, utilizando para tanto um pigmento diferente do original. Outro detalhe interessante refere-se à amostra 9, obtida do interior do quadro, a qual também apresentou espectro Raman igual às demais amostras, denotando uma vez mais que Portinari muito provavelmente utilizou azul ultramarino como coloração básica de fundo para a confecção da obra.

Com relação aos espectros obtidos para a amostra 2 , podese dizer que o emassamento, referente ao padrão observado para o pigmento azul da Prússia, é uma cobertura sobre o pigmento original, que apresenta o padrão espectral observado para as demais partes investigadas do quadro.

Com relação à datação da obra, cabe lembrar mais uma vez que o mesmo não apresenta, nas amostras de 3 a 10, a presença de óleo dispersante para o pigmento, indicando que a obra foi realizada por Portinari há pelo menos 50 anos, coincidindo com a data observada abaixo da assinatura do autor, referente a 1933 
ou 1935. A parte emassada com pigmento à base de azul da Prússia, mais recente, ainda apresenta uma considerável parcela de dispersante presente na estrutura do pigmento.

\section{CONCLUSÕES}

Em função das medidas de microscopia Raman efetuadas nas regiões da obra assinaladas na figura 1, pode-se afirmar que um dos pigmentos originais utilizados por Cândido Portinari no quadro a óleo "Retrato de Murilo Mendes" foi o lápis lazuli, ou seu correspondente sintético azul ultramarino. A presença de outros pigmentos não foi detectada nas regiões investigadas. $\mathrm{Na}$ parte do quadro que sofreu interferência posterior, foi utilizado como pigmento para emassamento o azul da Prússia, e a presença de bandas Raman referentes a compostos orgânicos fornece subsídios para supor que esta interferência foi muito mais recente do que a data de realização da obra original.

\section{AGRADECIMENTOS}

Os autores agradecem ao CNPq pelas bolsas concedidas, à FAPESP pela concessão da instrumentação utilizada, e ao
Centro de Estudos Murilo Mendes da Universidade Federal de Juiz de Fora pela permissão para utilização da obra, pertencente a seu acervo. Os autores também agradecem ao Prof. Henrique E. Toma pela gentil cessão de uma amostra de azul da Prússia.

\section{REFERÊNCIAS}

1. Clark, R. J. H.; Chem. Soc. Rev. 1995, 118, 187.

2. Ciomartan, D. A. e Clark, R. J. H.; J. Braz. Chem. Soc. 1996, 7, 395

3. Nakamoto, K., "Infrared and Raman Spectra of Inorganic; and Coordination Compounds", Wiley, fourth ed., New York, 1986.

4. Clark, R. J. H. e Franks, M. L.; Chem. Phys. Lett. 1975, $34,69$.

5. Clark, R. J. H. e Cobbold; D. G.; Inorg. Chem. 1978, 17, 3169.

6. Clark, R. J. H., Dines, T. J e Kurmoo, M.; Inorg. Chem. 1983, 22, 2766.

7. Butler, A. R. e Gash, R.; Chem. Br. 1993, 952.

8. Boscán, J. C. R. P., informação pessoal. 